\title{
Collective Formation and Cooperative Function of a Magnetic Microrobotic Swarm
}

\author{
Xiaoguang Dong, Metin Sitti \\ Max Planck Institute for Intelligent Systems, Stuttgart 70569, Germany. \\ Email: xdong@is.mpg.de; sitti@is.mpg.de
}

\begin{abstract}
Untethered magnetically actuated microrobots can access distant, enclosed and small spaces, such as inside microfluidic channels and the human body, making them appealing for minimal invasive tasks. Despite the simplicity of individual magnetic microrobots, a collective of these microrobots that can work closely and cooperatively would significantly enhance their capabilities. However, a challenge of realizing such collective magnetic microrobots is to coordinate their formations and motions with underactuated control signals. Here, we report a method that allows collective magnetic microrobots working closely and cooperatively by controlling their two-dimensional (2D) formations and collective motions in a programmable manner. The actively designed formation and intrinsic adjustable compliance within the group allow bio-inspired collective behaviors, such as navigating through cluttered environments and reconfigurable cooperative manipulation ability. These collective magnetic microrobots thus could enable potential applications in programmable self-assembly, modular robotics, swarm robotics, and biomedicine.
\end{abstract}

Keywords- swarm; magnetic microrobots; bio-inspired; cooperation; collective behavior.

\section{INTRODUCTION}

Untethered mobile miniature robots without on-board actuators and power sources could be actuated by external stimuli, such as light, acoustics, and electrostatic and magnetic fields [1]. In particular, mobile microrobots actuated and controlled by external magnetic fields can access distant, enclosed and small spaces, such as inside microfluidic channels and the human body, making them appealing for minimally invasive applications, such as biomedicine [2-4]. While single magnetic miniature robots have already shown promising functionalities [2-6], their simplicity raises considerably when scaling down to submillimeter- and micro-scale, limiting their capabilities. Despite the simplicity of individual magnetic microrobots, a collective of these microrobots that can work closely and cooperatively would significantly enhance their functionalities. When equipped with advanced programmability of formations and collective motions, these collective magnetic microrobots could also have unprecedented applications in programmable self-assembly [7], swarm robotics [8, 9], and modular robotics [10].

However, enabling a large number of magnetic microrobots to work cooperatively is challenging because magnetic microrobots cluster easily due to magnetic interactions and their individual motions are highly coupled. Many methods have been proposed aiming at controlling magnetic microrobots to work closely and cooperatively in large numbers [11], e.g., as microrobotic swarms, with programmable and reconfigurable functions. For example, one method is to decouple the motions of multiple heterogeneous microrobots by planning their dynamic motions with global actuation signals [12-14], where a small number of magnetic microrobots (up to three) have been independently controlled. Another strategy is to sequentially and spatially select specified microrobots to move, while anchoring other robots by auxiliary forces such as electrostatic forces [15] or magnetic gradient pulling forces [16]. Other works include simultaneously assembling ferromagnetic microrobots [17-21] into simple formations and structures, as well as actuating paramagnetic helix swarms [22, 23], magneto-bacteria clusters [24, 25], and paramagnetic microparticles or nanoparticles in formations of asters [26] and ribbons [27]. Although these existing methods all represent advancements, they still cannot produce versatile desired formations and reconfigurable cooperative functions among a large number of magnetic microrobots.

Here, we report a generic methodology of producing desired two-dimensional (2D) formations and bio-inspired collective and cooperative behavior among collective ferromagnetic microrobots (from $\sim 100 \mu \mathrm{m}$ to $\sim 350 \mu \mathrm{m}$ in diameter and up to $\sim 260$ in number, referred as microrobots by convention). Our method is to program the system equilibrium of collective selfrepelling magnetic microrobots by patterning desired magnetic potential energy maps (Fig. 1). The method is potentially also applicable on 2D solid substrates or with other type of physical constraints, but we focus on the air-water interface in this work as a proof of concept. With the proposed method, versatile complex formations were created among collective magnetic microrobots. Inspired by the collective and cooperative behavior of animals [28, 29], we further demonstrated constructing microrobotic swarms capable of bio-inspired collective behavior such as collective navigation through complex environments by adapting to the environmental morphological constraints, and reconfigurable cooperative manipulation of objects in different numbers and shapes.

\section{CONCEPT}

\section{A. Collective formation}

The key concepts of programming static formations include two parts. First, to avoid magnetic robots clustering when close 


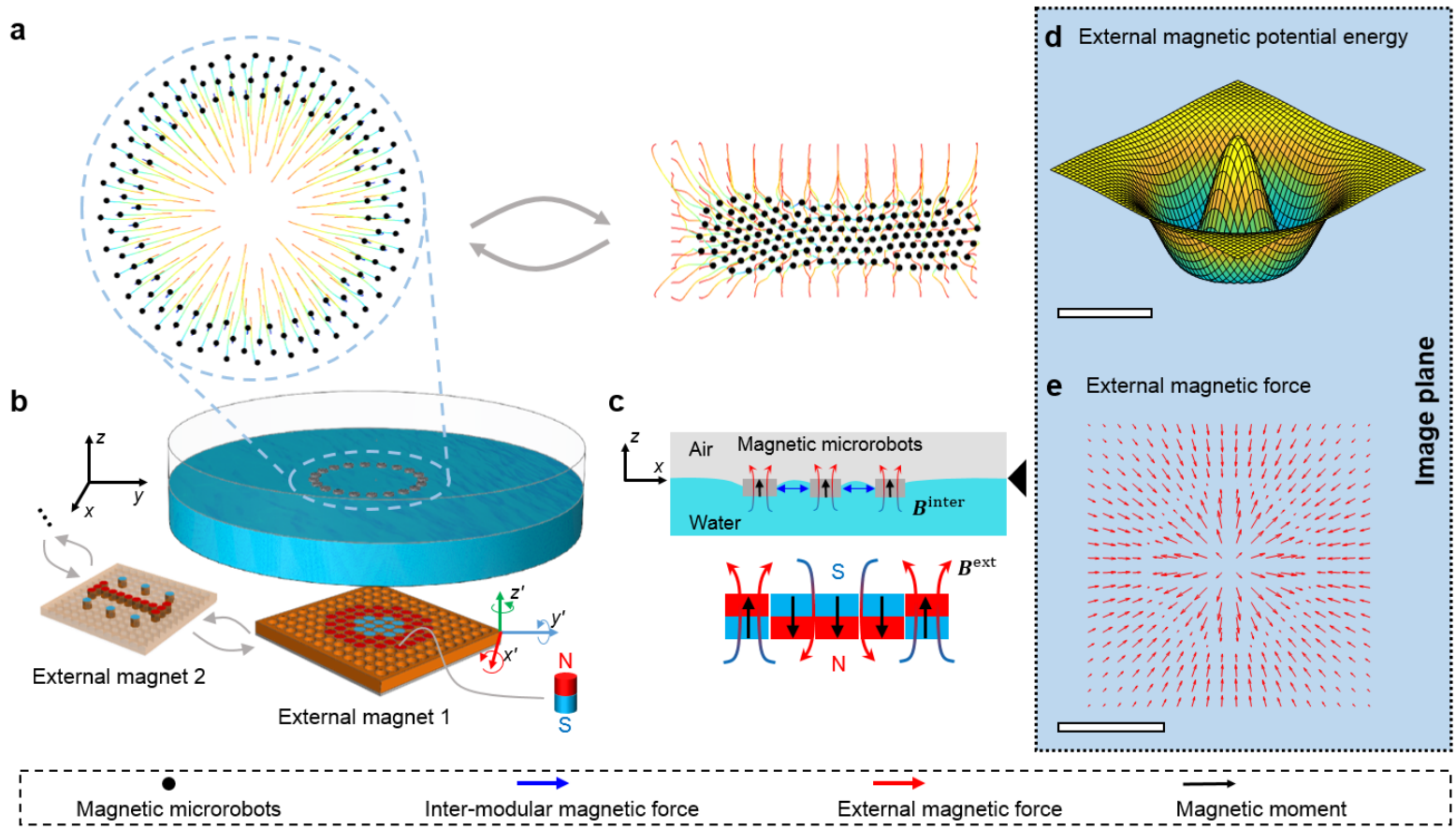

Fig. 1. Concept and the designed programmable collective system. (a), Concept of programmable and reconfigurable formation of collective magnetic microrobots. Black dots and colored lines represent magnetic microrobots and their trajectories, respectively. (b), Overview of the designed programmable collective system. Programmed external magnets induce various pattern formations of ferromagnetic microrobots at the air-water interface. The external magnet can translate and rotate in three-dimensional space for producing time-varying formations and collective motions. (c), Side of the designed programmable collective system. Ferromagnetic microrobots with vertical magnetic moments repel each other due to inter-modular repulsive magnetic forces. The external magnet creates spatially variant magnetic fields $\boldsymbol{B}^{\text {ext }}$ superimposed with inter-modular magnetic fields $\boldsymbol{B}^{\text {inter }}$. Surface tension on the image plane balances the forces along the $z$ axis and rigid-body tilting torques. (d), Modeled external magnetic potential energy map on the image plane for a ring-shaped formation. (e), Modeled external magnetic force distribution on the image plane for a ring-shaped formation. Scale bars: $5 \mathrm{~mm}$.

to each other, their motions must be constrained such that their inter-modular reaction is always repulsive. Second, the equilibrium positions of these microrobots must be programmable. Here, we propose to create collective selfrepelling magnetic microrobots and program their equilibrium positions by designing an external magnetic potential energy distribution. The air-water interface (Fig. 1b) is employed as a physical constraint in this work as a proof of concept, although the method is potentially also applicable on 2D solid substrates, which will be discussed briefly in the discussion section. The air-water interface allows surface tension to constrain the orientations of magnetic microrobots vertically and balance forces in the $z$ direction, therefore, magnetic microrobots avoid clustering into each other due to the inter-modular repulsive magnetic forces (Fig. 1c). For multiple magnetic microrobots exposed to an external magnetic field $\boldsymbol{B}^{\text {ext }}$ produced by an external magnet, the magnetic potential energy $U$ of the $i$-th magnetic microrobot at a position $\boldsymbol{r}_{i}$ with a magnetic moment $\boldsymbol{M}_{i}$ is a superposition of the potential energy resulting from both external and inter-modular magnetic fields so that

$$
U\left(\boldsymbol{M}_{i}, \boldsymbol{r}_{i}\right)=U^{\text {inter }}\left(\boldsymbol{M}_{i}, \boldsymbol{B}^{\text {inter }}\left(\boldsymbol{r}_{i}\right)\right)+U^{\operatorname{ext}}\left(\boldsymbol{M}_{i}, \boldsymbol{B}^{\text {ext }}\left(\boldsymbol{r}_{i}\right)\right) .
$$

With the constraint at the air-water interface, the magnetic potential energy from the inter-modular interaction $U^{\text {inter }}$ is negligible compared with that from the external magnetic field $U^{\text {ext }}$, when the microrobots are far from each other. All the microrobots will move towards a position with a local or global minimum of the external magnetic potential energy $U^{\text {ext }}$. When they approach to each other, the inter-modular magnetic potential energy $U^{\text {inter }}$ increases and modifies the total magnetic potential energy distribution. Finally, at an equilibrium state, magnetic microrobots transform to a static pattern to minimize the total magnetic potential energy $\sum_{i=1}^{J} U_{i}\left(\boldsymbol{M}_{i}, \boldsymbol{r}_{i}\right)$ (J: number of robots). Individual equilibrium positions of magnetic microrobots depend on both their initial positions and the distribution of the total magnetic potential energy. At submillimeter scale, independently controlling individual agents is difficult due to the severe under-actuation of external magnetic fields. Instead of independently addressing each microrobot to a specific position, we propose to concurrently control the overall formation of the magnetic microrobots by programming $U^{\text {ext }}$ spatially.

As an illustrative example, to create a ring-shaped formation, we produced an external magnetic potential energy landscape on the image plane as shown in Fig. 1d, of which the spatial gradient is the desired external magnetic force (Fig. 1e). The achieved ring-shaped formation (Supplementary Movie 
S1) of microrobots minimized the total magnetic potential energy of the system.

\section{B. Soft-body like collective motion}

When collective magnetic microrobots reach the system equilibrium, they transform into a spring network with a specific morphology, although each microrobot is not physically connected. The whole group as a spring-network has an unconventional soft-body like behavior, such as collective mobility when maintaining formation, and intrinsic adjustable compliance. Such soft-body like collective magnetic modules as a microrobotic swarm thus allow collective behaviors like animal collectives and adaptability and flexibility when interacting with environments and objects.

\section{THEORY AND METHOD}

This section presents the theory and method of programming static formations and controlling collective motions.

\section{A. Dynamics of collective magnetic microrobots}

The collective system can be modeled as a mass-springdamper network. The 2D dynamics of each microrobot at time $t$ is given by,

$$
\begin{gathered}
m_{i} \frac{d^{2} \boldsymbol{r}_{m i}}{d t^{2}}=\boldsymbol{F}_{\boldsymbol{i}}^{\text {mag,ext }}\left(\boldsymbol{r}_{m i} ; \boldsymbol{r}_{\boldsymbol{a}}(t), \boldsymbol{M}_{\boldsymbol{a}}(t)\right)+\sum_{j=1, j \neq i}^{J}\left[\boldsymbol{F}_{i j}^{\text {mag,inter }}\left(\boldsymbol{r}_{m i}, \boldsymbol{r}_{m j}\right)+\right. \\
\left.\boldsymbol{F}_{i j}^{\text {capillary }}\left(\boldsymbol{r}_{m i}, \boldsymbol{r}_{m j}\right)\right]+\boldsymbol{D} \frac{d \boldsymbol{r}_{m i}}{d t},
\end{gathered}
$$

where $i=1, \ldots, J, m_{i}$ and $\boldsymbol{r}_{m i}$ are the mass and position of the $i$-th magnetic microrobot, respectively. $\boldsymbol{D}$ is the $2 \mathrm{D}$ drag force coefficient matrix (assuming at low Reynolds number), $\boldsymbol{F}^{\text {mag,ext }}\left(\boldsymbol{r}_{m i}, t\right)=\frac{\partial U^{\text {mag,ext }}(t)}{\partial \boldsymbol{r}_{m i}}$ is the external magnetic force exerting on the $i$-th magnetic microrobot which depends on the positions $\boldsymbol{r}_{\boldsymbol{a}}(t)$ and magnetic moments $\boldsymbol{M}_{\boldsymbol{a}}(t)$ of external magnets, and $\boldsymbol{F}_{i j}^{\text {mag,inter }}$ and $\boldsymbol{F}_{i j}^{\text {capillary }}$ are the interactive magnetic force and horizontal capillary force applied from the $j$-th to the $i$-th magnetic microrobot, respectively. The model assumes the forces in the vertical direction have balanced, and the motion of each magnetic microrobot emerges from horizontal magnetic and capillary forces while damped by the fluid drag.

\section{B. Static formation as system equilibrium}

At a static state, the overall formation depends on the system equilibrium under a spatially distributed external magnetic field. The system equilibrium can be derived from the total potential energy, which is given by

$$
\begin{array}{r}
U\left(\boldsymbol{r}_{m}, \boldsymbol{o}_{\boldsymbol{m}} ; \boldsymbol{r}_{a}, \boldsymbol{M}_{a}\right)=\frac{1}{2} \sum_{i=1}^{J} \sum_{j=1, j \neq i}^{J}\left[-\boldsymbol{M}_{m i} \cdot \boldsymbol{B}_{i j}^{\mathrm{inter}}\left(\boldsymbol{r}_{m i}, \boldsymbol{r}_{m j}, \boldsymbol{M}_{m j}\right)\right] \\
+\sum_{i=1}^{J} \sum_{k=1}^{N}\left[-\boldsymbol{M}_{m i} \cdot \boldsymbol{B}_{a, k i}^{\mathrm{ext}}\left(\boldsymbol{r}_{m i} ; \boldsymbol{r}_{a k}, \boldsymbol{M}_{a k}\right)\right]+U_{o}\left(\boldsymbol{r}_{\boldsymbol{m}}, \boldsymbol{o}_{\boldsymbol{m}}\right)
\end{array}
$$

Here, $\boldsymbol{r}_{m}, \boldsymbol{o}_{m}$ (concatenated 2D position and orientation vectors of magnetic microrobots) are the states of the network and $\boldsymbol{r}_{a}, \boldsymbol{M}_{a}$ (concatenated position and magnetic moment vectors of external magnets) are the parameters of the network, $\boldsymbol{B}_{i j}^{\text {inter }}$ and $\boldsymbol{B}_{a, k i}^{\text {ext }}$ represent magnetic fields applied on the $i$-th magnetic microrobot (with a magnetic moment $\boldsymbol{M}_{m i}$ ) at the position $\boldsymbol{r}_{m i}$ by the $j$-th magnetic microrobot and the $k$-th external magnet, and $U_{o}\left(\boldsymbol{r}_{\boldsymbol{m}}, \boldsymbol{o}_{\boldsymbol{m}}\right)$ is other type of potential energy, which is assumed to be constant. The system equilibrium is thus given by minimizing the total potential energy as,

$$
\begin{gathered}
\boldsymbol{F}_{m}^{\text {net }}=\frac{\partial U\left(\boldsymbol{r}_{\boldsymbol{m}}, \boldsymbol{o}_{\boldsymbol{m}} ; \boldsymbol{r}_{\boldsymbol{a}}, \boldsymbol{M}_{\boldsymbol{a}}\right)}{\partial \boldsymbol{r}_{\boldsymbol{m}}}=\mathbf{0}, \\
\boldsymbol{K}_{m}^{\text {net }}=\frac{\partial^{2} U\left(\boldsymbol{r}_{\boldsymbol{m}}, \boldsymbol{o}_{m} ; \boldsymbol{r}_{\boldsymbol{a}}, \boldsymbol{M}_{\boldsymbol{a}}\right)}{\partial \boldsymbol{r}_{\boldsymbol{m}}^{2}} \geq \mathbf{0} .
\end{gathered}
$$

If we look at the system equilibrium regarding individual positions of these magnetic microrobots, the system equilibrium does not have a unique solution because swapping the individual positions of microrobots doesn't change the total magnetic potential energy. Instead of directly designing the system equilibrium, we design the overall formation via the external magnetic potential energy distribution in the global Eulerian space.

\section{Programing static formation}

The system equilibrium can be controlled by the external magnetic potential energy distribution $U^{\text {ext }}(\boldsymbol{r})$. This allows controlling the overall formation rather than individual positions of microrobots. Furthermore, we can equivalently program an external magnetic field $\boldsymbol{B}^{\text {ext }}(\boldsymbol{r})$ spatially, assuming the magnetic moment of each microrobot is vertical and invariant of its position. The desired external magnetic field $\boldsymbol{B}^{\text {ext }}(\boldsymbol{r})$ at a position $\boldsymbol{r}$ can be further approximated with $N$ external magnetic sources $\boldsymbol{B}^{\operatorname{ext}}(\boldsymbol{r})=$ $\sum_{k=1}^{N} \boldsymbol{B}_{a k}\left(\boldsymbol{r} ; \boldsymbol{r}_{a k}, \boldsymbol{M}_{a k}\right)$, where each external magnet has a position $\boldsymbol{r}_{a k}$ and a magnetic moment $\boldsymbol{M}_{a k}$. As one approach, programming ferromagnets will be explained in Section IV.

\section{Controlling collective motion}

On top of static formations, with a spatiotemporally varying external magnetic potential energy distribution $\boldsymbol{U}^{\text {mag,ext }}(\boldsymbol{r}, \boldsymbol{t})$, collective motion can be further controlled. For example, magnetic microrobots can aggregate into a compact formation, by decreasing the size and increasing the strength of the external magnetic potential well. Moreover, magnetic microrobots could translate and turn in a desired trajectory while still maintaining their formation shape, by translating the potential energy distribution in the $x-y$ plane and rotating it about its $z$ ' axis (in the body frame). These collective behavior will be shown in Section VII.

\section{PROGRAMming EXTERnAl Magnets}

As one approach, we encoded the external magnetic potential energy distributions with multiple modular ferromagnets with magnetic moments in discrete values. Compared with electromagnets [30], ferromagnets can produce a more complex magnetic potential energy distribution in a longer range. Existing work on programming ferromagnets have been mainly focusing on producing uniform magnetic fields using optimized Halbach arrays [31, 32]. Here we focused on programming ferromagnets for a desired nonuniform distribution of magnetic fields and magnetic potential energy. 

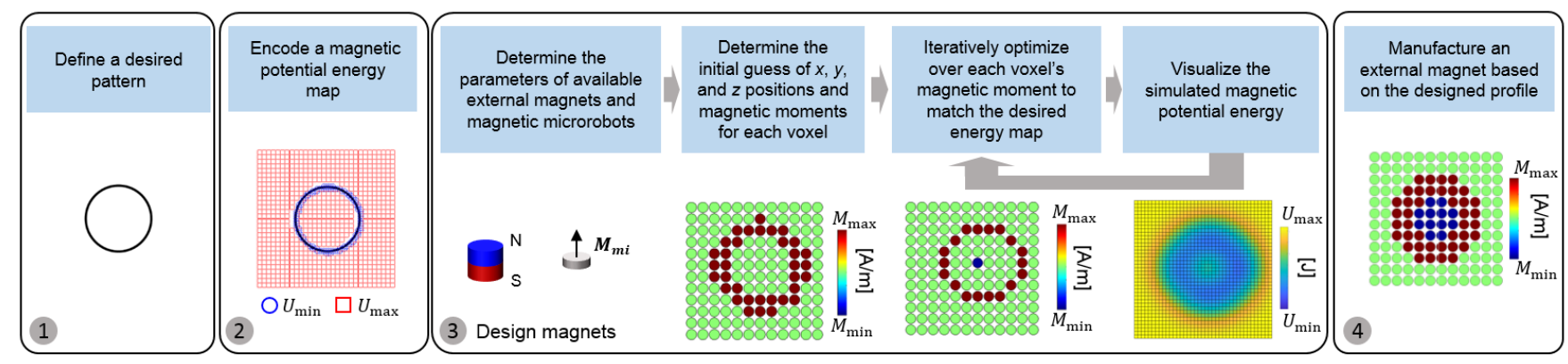

Fig. 2. Programming external magnetic potential energy maps using external magnets with discrete magnetization profiles. Overall workflow of designing an external magnet array for a desired external magnetic potential energy map given a user-defined pattern. In the color bars: $U_{\min }=-1.60 \times 10^{-9} \mathrm{~J}$ and $U_{\max }=5.19 \times 10^{-10} \mathrm{~J} ; M_{\min }=-735 \mathrm{kA} \mathrm{m}^{-1}$ and $M_{\max }=735 \mathrm{kA} \mathrm{m}^{-1}$.

The desired magnetic potential energy distribution was matched by programming the positions $\boldsymbol{r}_{a}(3 \times N$ matrix, concatenated by the position vector $\boldsymbol{r}_{a k}$ of each external magnet) and numbers $\boldsymbol{N}_{a}\left(N \times 1\right.$ vector, $N_{a k} \in\{-1,0$, or 1$\left.\}\right)$ of $N$ discrete external modular or bulk-shaped ferromagnets. Here "0" means there is no magnet, and "1" or "- 1 " indicates an upward or downward magnetic moment. The problem is given by,

$$
\min _{\boldsymbol{r}_{a}, \boldsymbol{N}_{\boldsymbol{a}}} \sum_{i=1}^{P}\left\|\bar{U}^{\mathrm{mag}, \mathrm{ext}}\left(\boldsymbol{r}_{m i}\right)+\boldsymbol{M}_{m i} \cdot \boldsymbol{B}\left(\boldsymbol{r}_{a}, \boldsymbol{r}_{m i}\right) \cdot \boldsymbol{N}_{a}\right\|,
$$

where $\bar{U}^{\text {mag,ext }}\left(\boldsymbol{r}_{m i}\right)$ is the targeted external magnetic potential energy of a microrobot with a magnetic moment vector $\boldsymbol{M}_{m i}$ at the position $\boldsymbol{r}_{m i}(P$ sampled points in total), and $\boldsymbol{B}$ is a $3 \times N$ matrix mapping the number of discrete magnets $\boldsymbol{N}_{\boldsymbol{a}}$ to the magnetic field at $\boldsymbol{r}_{m i}$. The general programming steps are explained as below (Fig. 2).

a) The desired magnetic potential energy distribution was encoded from the desired formation pattern. The energy of a desired pattern was set as negative values, which scale with the magnetic moments of external magnets and magnetic microrobots, and the distance from external magnets to the image plane. Energy of other parts were set as zero.

b) The potential positions of modular or bulk-shaped magnets were sampled in a predefined three-dimensional (3D) volume, which is usually under the image plane and has similar sizes with the formation pattern for $x$ and $y$ dimensions.

c) The magnetic moment at each potential position was iteratively optimized by choosing the number of modular magnets $N_{a k}(-1,0$, or 1$)$ at a sampled position.

d) A finite-element model (Multiphysics 5.3a and Livelink for MATLAB, COMSOL Inc.) of ferromagnets was used for computation of the external magnetic potential energy based on the iteratively designed magnetization profile in c). Then, Step c) was revisited to iteratively optimize the magnetization profile for matching the desired potential energy distribution. The procedure from Step c) to Step d) typically took 5 to 10 iterations for the formations demonstrated in this paper. A high-dimensional integer-optimization algorithm [33,34] can potentially be incorporated for the Steps c) and d) to automatically optimize the magnetization profile. The magnetic dipole model can replace the finite-element model for more efficient computation when the distances from external magnets to the image plane are relatively long compared with the size of an individual ferromagnet [35].

e) The formations of magnetic microrobots were simulated based on the obtained magnetic potential energy distribution in d) and the predictive dynamic model in Eqn. (2).

\section{EXPERIMENTAL SETUP}

This section presents the physical implementation of programming external magnets using discrete ferromagnets, the preparation and characterization of the magnetic microrobots, and experimental procedures.

\section{A. Assembling external ferromagnets}

Discrete ferromagnets were assembled into wood jigs for fixing their positions and orientations according to the designed profile. The wood jigs were cut by a laser cutting machine (ProtoLaser 3D, LPKF Laser \& Electronics AG) with an accuracy of $\pm 25 \mu \mathrm{m}$ in $x$ and $y$ dimensions. Adhesive tapes (Tesa SE) were attached at the bottom of the jigs for fixing the $z$ positions of these magnets. The discrete ferromagnets assembled were commercial $\mathrm{NdFeB}$ magnets (Supermagnete.de) in cylindrical and cubic shapes.

\section{B. Fabrication of magnetic microrobots}

The magnetic microrobots were made of microparticles of neodymium-iron-boron (NdFeB) alloy (MQP-15-7, Magnequench, Inc.; average diameter: $5 \mu \mathrm{m}$, density: 7.61 $\mathrm{g} / \mathrm{cm}^{3}$, magnetic relative permeability: 1.05 ) and casting resins (Cast 310, Smooth-On, Inc.; density: $1.05 \mathrm{~g} / \mathrm{cm}^{3}$ ) with $20 \mathrm{wt} \%$ to $50 \mathrm{wt} \%$ of $\mathrm{NdFeB}$ magnetic microparticles. $\mathrm{NdFeB}$ microparticles were selected for fabricating magnetic microrobots because they have high remanent magnetization for exhibiting strong magnetic interaction and large magnetic coercivity for keeping such a magnetization. We chose cast resin because of its low density and large water contact angles for better floating of magnetic microrobots at the air-water interface. These two materials were mixed together and casted into a Polydimethylsiloxane (PDMS) mold obtained from a two-step photolithography molding method. The magnetic microrobots were molded in thin disk shapes. The top surface of the magnetic material was scraped with a razor carefully, which may cause a convex shape on the top surface. After 
polymerization, all these magnetic microrobots in disk shapes were magnetized perpendicular to their top surfaces by a $1.0 \mathrm{~T}$ to $1.6 \mathrm{~T}$ uniform magnetic field generated by a vibrating sample magnetometer (EZ7 VSM, Microsense, LLC).

\section{Characterization of magnetic microrobots}

The magnetic microrobots in disk shapes in all the demonstrations had an average diameter of $350.5 \mu \mathrm{m} \pm 4.0 \mu \mathrm{m}$ $(n=5)$ and an average thickness of $100.2 \mu \mathrm{m} \pm 20.0 \mu \mathrm{m}(n=$ $5)$. The static water contact angles were measured using the sessile droplet method through an automated goniometer routine (DSA100, Krüss GmbH). For the magnetic composite material with $20 \mathrm{wt} \% \mathrm{NdFeB}$, the static water contact angles were measured as $\theta_{a}=95^{\circ} \pm 3^{\circ}, \theta_{r}=65^{\circ} \pm 6^{\circ}(n=3)$ for the back surface, and $\theta_{a}=98^{\circ} \pm 2^{\circ}, \theta_{r}=70^{\circ} \pm 4^{\circ}(n=3)$ for the top surface. For the magnetic composite material with $50 \mathrm{wt} \%$ $\mathrm{NdFeB}$, the static water contact angles were measured as $\theta_{a}=$ $96^{\circ} \pm 2^{\circ}, \theta_{r}=69^{\circ} \pm 5^{\circ}(n=3)$ for the back surface, and $\theta_{a}=$ $95^{\circ} \pm 2^{\circ}, \theta_{r}=67^{\circ} \pm 6^{\circ}(n=3)$ for the top surface. The fabricated magnetic microrobots were not subjected to any treatment before the experiments. The magnetic moments of magnetic microrobots were measured in a vibrating sample magnetometer (EZ7 VSM, Microsense, LLC). The magnetic moment density (magnetization) was then obtained by dividing the corresponding volumes measured by a laser scanner (VKX200, Keyence Corp.).

\section{Experimental procedure.}

The experimental setup comprised a petri-dish container in two different sizes (inner diameters: $137 \mathrm{~mm}$ and $89 \mathrm{~mm}$; height: $18 \mathrm{~mm}$ and $14 \mathrm{~mm}$, respectively) filled with deionized water. The containers were supported by customized sample holders. Magnetic microrobots were manually put at the airwater interface by tweezers with the back surface touching water. This procedure was assisted by an external ferromagnet under the container. The distance from the air-water interface to the bottom of the container was adjusted by adding or retrieving water. An artificial arena was also added inside the bigger petri-dish. In the experiments, external magnets were manually translated and rotated to produce spatiotemporal external magnetic potential energy maps, which could be replaced by an $x-y-z-\theta$ motorized stage or a robotic arm in the future.

\section{RESULTS: FORMATION}

\section{A. Static formations}

In Fig. 3, we demonstrated creating a variety of desired static formations among 146 magnetic microrobots at the airwater interface, including letters "M", "P", "I", "A", "B", "C" and a "wrench"-shaped pattern (Fig. 3a and Supplementary Movie S1). The versatility of formations arises from the programmability of external magnetic potential energy maps. To create the desired external magnetic potential maps, we programmed arrays of cubic or cylindrical modular ferromagnets as external magnets (Fig. 3b). Predictive models of magnetic potential energy maps and collective formations

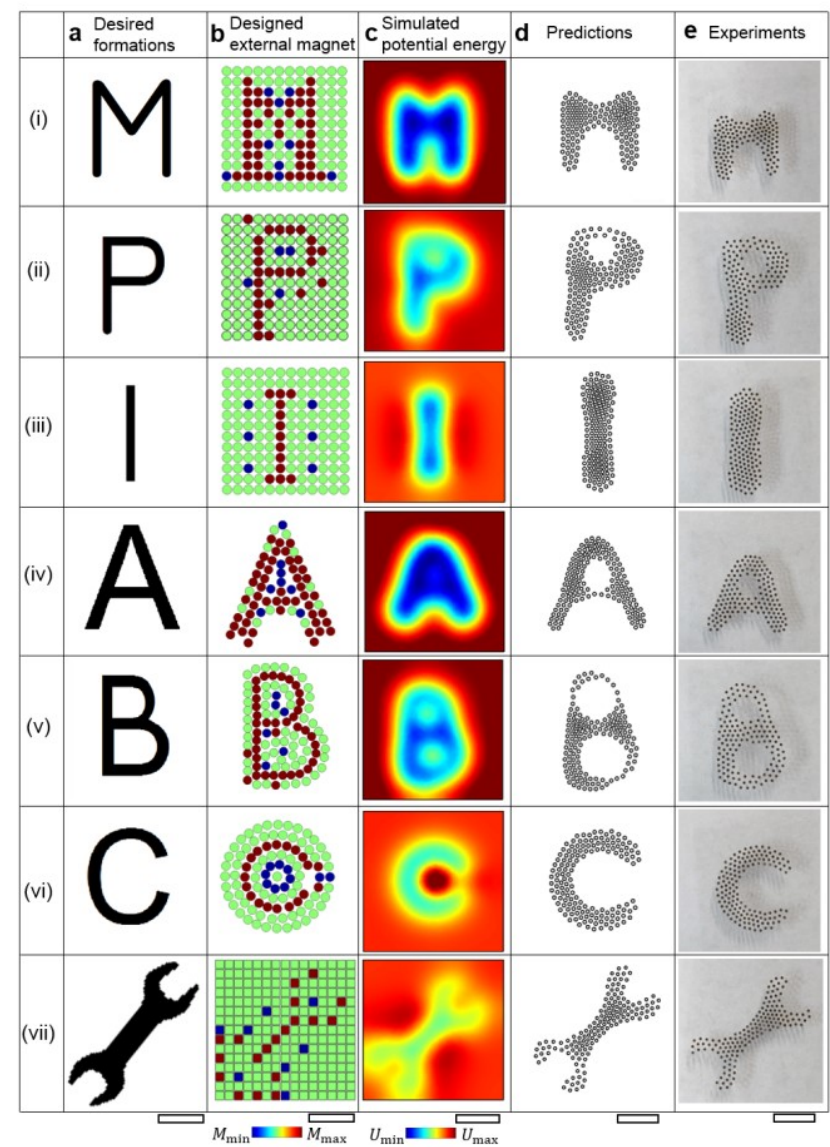

Fig. 3. Experimentally realized static formations of collective magnetic microrobots, demonstrating the programming method. (a), Desired formation patterns. (b), Magnetization profiles of designed external magnets. The circular and square-shaped elements represent cylindrical and cubic magnets, respectively. The color represents the magnetization magnitude and direction. In the color bar: $M_{\min }=-735 \mathrm{kA} \mathrm{m}^{-1}$ and $M_{\max }=735 \mathrm{kA} \mathrm{m}^{-1}$. (c), Simulated magnetic potential energy from the interaction between a magnetic microrobot and the designed external magnets. In the color bar: $U_{\min }=-1.06 \times 10^{-9} \mathrm{~J}$ and $U_{\max }=3.54 \times 10^{-10} \mathrm{~J}$. (d), Predicted formations for 146 magnetic microrobots. Parameters used are same to that in experiments. (e), Video snapshots show experimentally realized static formation patterns of 146 magnetic microrobots at the air-water interface. The vertical distances from the top surfaces of the external magnets to the image plane are about $5 \mathrm{~mm}$ for "M", "P", "I", "C" and "wrench"-shaped formations and about $4 \mathrm{~mm}$ for " $A$ " and "B"-shaped formations in both simulations and experiments. All magnetic microrobots have an average diameter of $350 \mu \mathrm{m}$ and thickness of $100 \mu \mathrm{m}(20 \mathrm{wt} \% \mathrm{NdFeB}$ microparticles, magnetized in a $1.0 \mathrm{~T}$ magnetic field). Scale bars: $5 \mathrm{~mm}$.

were also used to guide the design of external magnets (Fig. 3c, d).

The proposed method allows both homogeneous and heterogeneous magnetic microrobots of various sizes and populations to form into desired $2 \mathrm{D}$ patterns. First, to create a full formation pattern, the population size of magnetic microrobots must be large enough to cover the region of interest (encoded by the desired pattern) in the potential energy map (Fig. 4a). Next, formations can be created with 


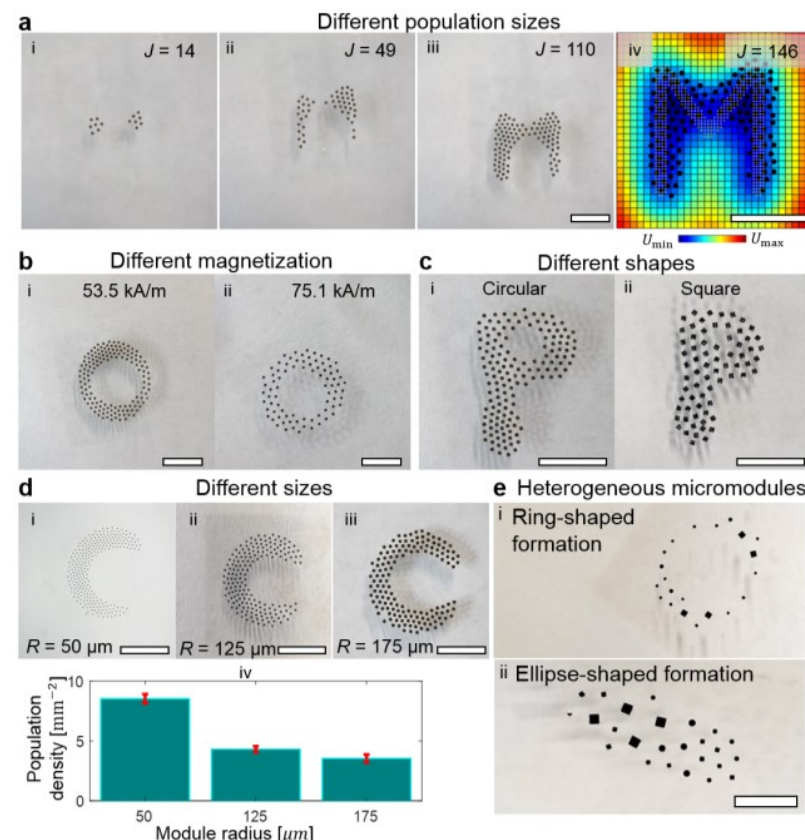

Fig. 4. Scaling analysis on static formations of magnetic microrobots. (a), Varying the population size (The number of magnetic microrobots $J$ is from 14 to 146) for an "M"-shaped formation until the region of interest (marked with yellow dots) on the potential energy map has been covered. Black dots in (iv) represent extracted positions of magnetic microrobots from an experimental image. In the color bar: $U_{\min }=-2.2 \times 10^{-9} \mathrm{~J}$ and $U_{\text {max }}=7.7 \times 10^{-10} \mathrm{~J}$. (b), Varying the magnetization of magnetic microrobots for a ring-shaped formation. i. 146 microrobots (magnetization: $53.5 \mathrm{kA} \mathrm{m}^{-1}$ ); ii. 81 microrobots (magnetization: $\left.75.1 \mathrm{kA} \mathrm{m}^{-1}\right)$. (c), Varying the shapes of magnetic microrobots for a "P"-shaped formation. i, 146 microrobots (disk shape, radius: $\sim 175$ $\mu \mathrm{m})$. ii, 68 microrobots (square shape, edge length: $\sim 500 \mu \mathrm{m}$ ). (d), Varying the sizes of magnetic microrobots for a " $\mathrm{C}$ "-shaped formation. i, 263 microrobots (disk shape, radius: $\sim 50 \mu \mathrm{m}$ ). ii, 239 microrobots (disk shape, radius: $\sim 125 \mu \mathrm{m}$ ). iii, 144 microrobots (disk shape, radius: $\sim 175 \mu \mathrm{m}$ ). iv, Population densities (robots number per area) of magnetic microrobots vs. microrobots sizes. Error bars indicate standard deviation of $n=3$ measurements for each case. (e), Formations with heterogeneous magnetic microrobots in circular, rectangular and square shapes for a ringshaped (i) and ellipse-shaped (ii) formation. Scale bars: $5 \mathrm{~mm}$.

homogeneous magnetic microrobots of various magnetic moments, sizes, and shapes. Exposed to the same external magnetic field, the formation packing density (microrobot number per unit area) is higher with magnetic microrobots of smaller magnetic moments (Fig. 4b) due to weaker intermodular repulsion. Formation pattern is independent of the sizes and shapes of magnetic microrobots but centrosymmetric shapes (Fig. 4c) and smaller sizes (Fig. 4d) are preferred for smoother contours of formations. Moreover, heterogeneous magnetic microrobots with different magnetic moments, shapes and other domain properties can also produce a desired formation (Fig. 4e). Finally, the formation stiffness is also programmable, allowing adaptive manipulation capability as microrobot collectives. The stiffness of a formation depends on the inter-modular distance and determines the compliance of
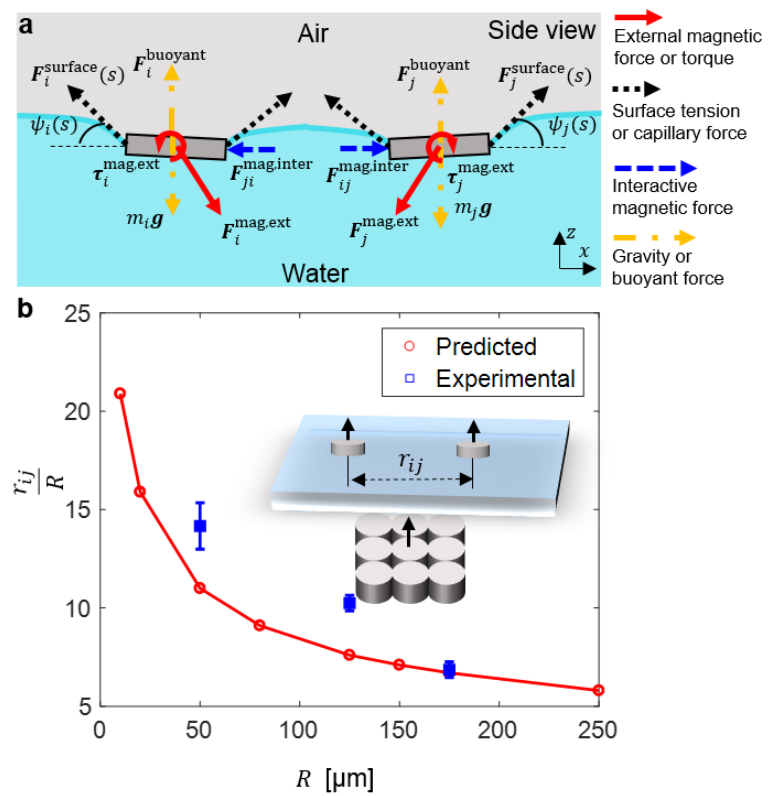

Fig. 5. Analysis of pair-wise interactions between magnetic microrobots. (a), Schematics of the pairwise interaction. (b), Predicted and experimental pair-wise equilibrium distances for magnetic microrobots in different diameters. Error bars indicate the standard deviation from $n=3$ measurements for each case. Magnetic microrobots have a magnetization of $50 \mathrm{kA} / \mathrm{m}$ and an aspect ratio of $\sim 33 \%$. Nine modular cylindrical magnets were placed under the image plane (distance: $8 \mathrm{~mm}$ ).

collective magnetic microrobots when moving together and interacting with other objects. Compared with existing methods of creating self-assembled magnetic structures [18-21] and other magnetic collectives [22-27], our method allows collective magnetic microrobots to have programmable and considerably more versatile formations simply by designing different external magnets.

\section{B. Pair-wise interaction at the air-water interface}

The pair-wise equilibrium distances for magnetic microrobots of different diameters were studied to understand the un-clustering conditions. Assuming the body axes and magnetic moments of microrobots are in vertical directions (Fig. 5a), the forces exerted on a pair of magnetic microrobots in the lateral directions are modeled and listed as follows,

a) Pairwise capillary force: $\boldsymbol{F}_{i j}^{\text {capillary }}=-\frac{2 \pi \gamma R^{2}(\sin \psi)^{2}}{\left|r_{i j}\right|^{2}} \boldsymbol{r}_{i j}$ (attractive), where $\gamma$ is the surface tension of water, and $\psi$ is the meniscal slope angle;

b) Pairwise inter-modular magnetic force: $\boldsymbol{F}_{i j}^{\text {mag,inter }}=$ $\frac{3 \mu_{0}}{4 \pi} \frac{\left|M \pi R^{2} h\right|^{2}}{\left|r_{i j}\right|^{5}} \boldsymbol{r}_{i j} \quad$ (repulsive), where $\mu_{0}$ is the magnetic permeability of vacuum, $h$ and $M$ are the thickness and magnetization of the microrobot;

c) External magnetic force: $\boldsymbol{F}_{i}^{\text {mag,ext }}=\boldsymbol{F}_{i}^{\text {mag,ext }}\left(\boldsymbol{r}_{m i} ; \boldsymbol{r}_{a}, \boldsymbol{M}_{a}\right)$.

Assuming $\psi$ is constant along the contact line for simplicity of scaling analysis, we have $\sin \psi=\frac{F_{z}}{2 \pi \gamma R}$ based on a monopole model [36], where $F_{z}$ is the capillary force in the $z$ 
direction. At an equilibrium state, the force balancing condition for the $i$-th (or $j$-th) magnetic microrobot is given by,

$$
\begin{aligned}
& \boldsymbol{F}_{i}^{\text {mag,ext }}\left(\boldsymbol{r}_{m i} ; \boldsymbol{r}_{a}, \boldsymbol{M}_{a}\right)= \\
& \quad-\sum_{j=1, j \neq i}^{J}\left[\boldsymbol{F}_{i j}^{\text {mag,inter }}\left(\boldsymbol{r}_{m i}, \boldsymbol{r}_{m j}\right)+\boldsymbol{F}_{i j}^{\text {capillary }}\left(\boldsymbol{r}_{m i}, \boldsymbol{r}_{m j}\right)\right]
\end{aligned}
$$

The inter-modular equilibrium distances must satisfy $\left|\boldsymbol{r}_{i j}\right|>$ $2 R$ to avoid touching or clustering of microrobots. Without losing generality, we analyzed the pairwise interaction between a pair of magnetic microrobots subject to an external magnetic field produced by nine modular external magnets under the image plane (distance: $\sim 8 \mathrm{~mm}$ ). As shown in Fig. $5 \mathrm{~b}$, both experimental results and dynamic simulation using the model in Eqn. (2) suggested that smaller magnetic microrobots would have a larger equilibrium distance ratio (the inter-modular distance over the radius of a microrobot).

\section{RESULTS: BIOINSPIRED FUNCTIONAL SWARM}

In this section, we further show a functional microrobotic swarm by demonstrating bio-inspired collective behaviors, such as collective navigation in cluttered environments and reconfigurable cooperative manipulation of objects.

\section{A. Soft-body like adaptive navigation}

Small animals can take advantage of their formation as a group for different tasks such as foraging, migrating, and escaping from danger [28]. For example, an army of fire ants [29] can survive from a water flood by collectively floating at the air-water-interface in a disk-shaped formation. In addition, soft-organisms [37] can navigate through cluttered environments exploiting their morphological adaptation and compliance when physically interacting with the environment.

Inspired by these behaviors, we created a microrobotic swarm capable of transforming into a formation and collectively navigating through confined spaces taking advantage of their actively designed formation and intrinsic compliance. The collective magnetic microrobots were capable of navigating through complex cluttered environments via morphology adaptation when interacting with the environmental boundaries (Fig. 6a and Supplementary Movie S2). First, the designed formation, such as an ellipse-shaped formation, provided a level of adaptation in morphology to the environmental boundaries by minimizing the contact area with channel walls. Moreover, the compliance from inter-modular repulsion further enhanced the adaptability and flexibility of negotiating through narrow channels and holes and over posts (Fig. 6b-d). For example, by pushing the boundaries and each other, magnetic microrobots could pass through narrow holes as shown in Fig. 6d. Finally, the redundancy in the collective microrobots also provides robustness for fault tolerance, similar to their counterparts in nature. For example, during the navigation, these magnetic microrobots still maintained their formation even when they lost some agents. Therefore, compared with a large monolithic robot, the designed microrobotic swarm have more flexibility of accessing confined space by adapting to the morphological constraints in the environments, and are more robust against agent failure.

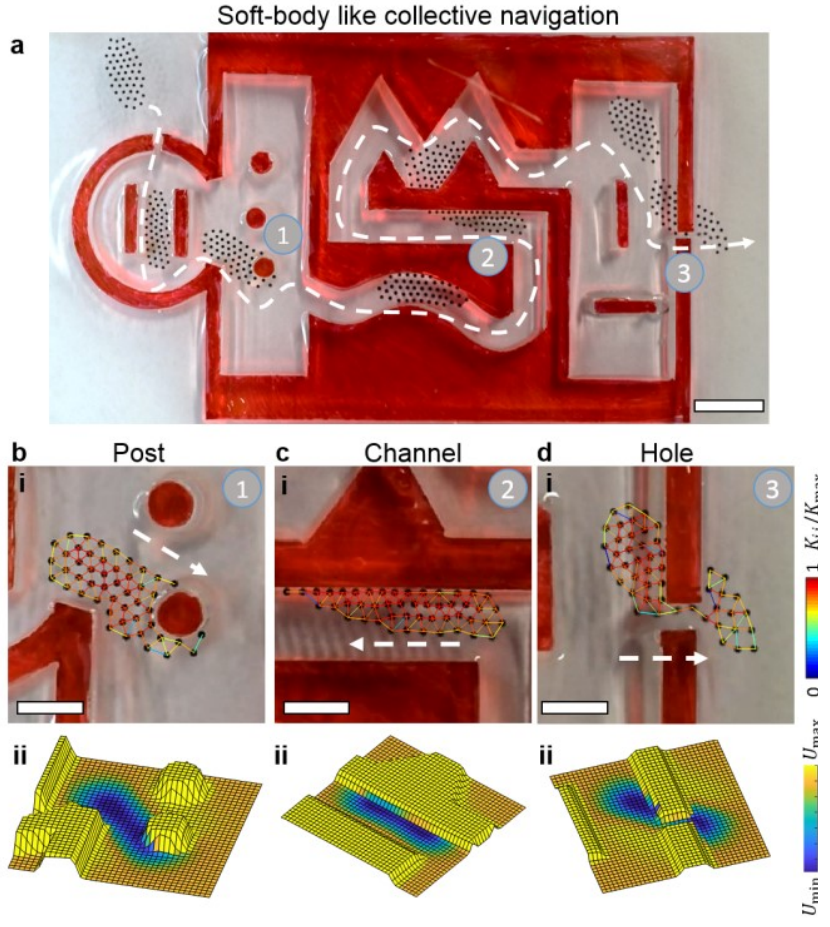

Fig. 6. Collective navigation of a microrobotic swarm through cluttered environments with morphological adaptation. (a), Video (Supplementary Movie 2) snapshots show 49 magnetic microrobots navigate in an ellipse-shaped formation in a cluttered environment. Locations marked with labels " 1 ", " 2 " and " 3 " are zoomed in (b-d). The dashed white arrows indicate the moving directions. (b-d). (i) Adaptation to environmental constraints from the inter-modular compliance of the microrobotic swarm while navigating around posts and through channels and holes and (ii) the modeled total potential energy maps from both external magnetic fields and environmental constraints (the environmental boundary energy was modeled using artificial potential field from [38]). Colored segments connecting magnetic microrobots in bd(i) indicate normalized inter-modular stiffness $K_{i j} \propto\left|\boldsymbol{r}_{i j}\right|^{-5}$ (5 nearest-neighbor connections are selected within a $5 R$ maximum Euclidian distance). In the color bars in b(ii), c(ii), and d(ii): $U_{\min }=-1.06 \times 10^{-9} \mathrm{~J}$ and $U_{\max }=1.06 \times 10^{-9} \mathrm{~J}$. All magnetic microrobots have an average diameter of $350 \mu \mathrm{m}$ and thickness of $100 \mu \mathrm{m}(50 \mathrm{wt} \% \mathrm{NdFeB}$, magnetized in a $1.2 \mathrm{~T}$ magnetic field $)$. Scale bars: $5 \mathrm{~mm}$.

\section{B. Reconfigurable cooperative manipulation}

Cooperative manipulation using collective magnetic matter has been shown before in micro-scale systems in experiments $[17,26,39,40]$ and simulations $[41,4]$, but they cannot complete versatile manipulation tasks due to the limited reconfigurability. In contrast, animal collectives can reconfigure their formations depending on the tasks, such as foraging and transportation, where the reconfiguration of formations allows diverse functions within the same group.

Inspired by such behaviors, in Fig. 7, we demonstrated cooperatively manipulating and transporting single and multiple objects in different shapes via pushing, grasping and caging (Supplementary Movie S3) manipulation strategies using the same collective magnetic microrobots. These 


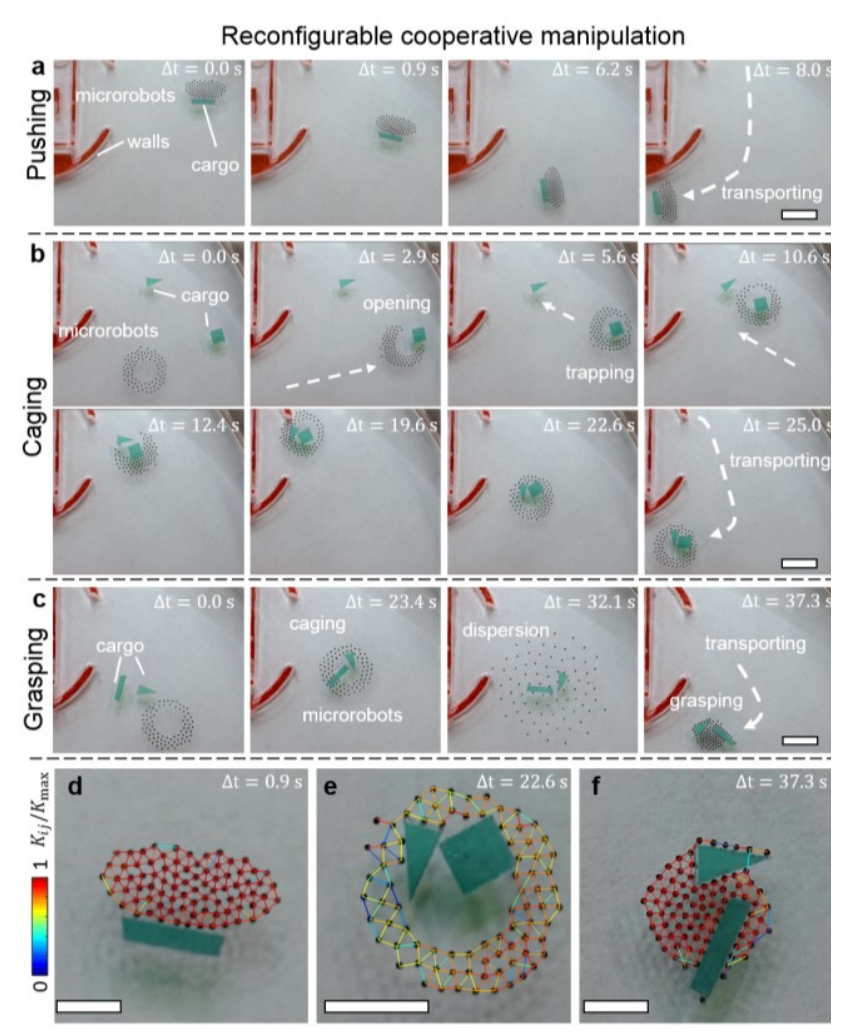

Fig. 7. Demonstrations of reconfigurable cooperative manipulation functions of a magnetic microrobotic swarm. (a), Video (Supplementary Movie 3) snapshots show collective transportation of an object in a long rectangular shape by 82 magnetic microrobots pushing in an ellipse-shaped formation. (b), Video (Supplementary Movie 3) snapshots show collective transportation of multiple objects by 82 magnetic microrobots caging in a ring-shaped formation. (c), Video (Supplementary Movie 3) snapshots show reliable grasping and transportation of multiple objects by 82 magnetic microrobots in a disk-shaped formation. (d-f). The inter-modular stiffness of 82 magnetic microrobots while transporting objects using pushing (d), caging (e) and grasping (f). Colored segments connecting magnetic microrobots in (d-f) indicate normalized inter-modular stiffness (5 nearest-neighbor connections are selected within a $5 R$ maximum Euclidian distance). All magnetic microrobots have an average diameter of $350 \mu \mathrm{m}$ and thickness of $100 \mu \mathrm{m}(50 \mathrm{wt} \% \mathrm{NdFeB}$, magnetized in a $1.2 \mathrm{~T}$ magnetic field). Scale bars: $5 \mathrm{~mm}$.

microrobots are capable of different on-demand formations depending on the task to be performed for an optimal performance. As shown in Fig. 7a, with an ellipse-shaped formation, the magnetic microrobots could uniformly and cooperatively push a long rectangular object and transport it to a desired location. The inter-modular stiffness of the formation was kept relatively high allowing collective microrobots maintaining the formation while transporting objects (Fig. 7d). After that, the same group of microrobots further transformed into a circular and more compliant formation, and adjusted the opening and closing states of the formation to trap and transport multiple objects gently and simultaneously (Fig. 7b, e). Sequentially transforming into circular and disk-shaped formations was then employed for demonstrating tight grasping of multiple objects with force closures (Fig. 7c). In this task, caging was firstly used to surround the targeted objects so that microrobots could disperse uniformly around the target objects. Then, a disk-shaped formation was engaged to aggregate these microrobots so that they could grasp objects in diverse shapes compactly and reliably as shown in Fig. 7f. The ability of reconfiguring into different formations with different morphologies and stiffness, i.e., tools, significantly enhanced the functionality of the collective magnetic microrobots. Here, we showed that the ability of reconfiguring different formation morphologies and stiffness on demand allows producing collective microrobots which are more functional and flexible, while being as effective as a larger taskspecific tool.

\section{CONCLUSION AND DISCUSSION}

In summary, this paper presented a generic methodology to program 2D formations of collective magnetic microrobots and demonstrated such a concept on submillimeter ferromagnetic modules at the air-water interface by programming discrete ferromagnet arrays. Despite the simplicity of the demonstrated system, remarkable programmability and reconfigurebility have been shown using the proposed methodology. In our demonstrations, although the individual magnetic moment and position of each ferromagnet in an array cannot vary on-site, we showed that the magnetic potential landscape can indeed be varied spatiotemporally by controlling the rigid body motions of external magnet arrays. Moreover, for formations on 2D solid substrates, a large uniform magnetic field can be applied to align magnetic microrobots vertically in addition to that produced by the designed external magnets, as the formation only depends on the spatial gradient of external magnetic field.

In addition to robotic applications, the proposed method and system can be potentially used for manufacturing, such as efficient 2D contactless material assembly [43]. Furthermore, the physical modular interaction in the collective system allows emulating animal-like behaviors, which may be helpful for researchers to use it as a platform to study swarm systems and animal behaviors [28] at small scales.

As future research directions, the actuation range is possible to be increased using stronger external magnets, for example, using superconducting materials [44] with efficient cooling in the extreme case. In addition, 3D formations is extremely challenging but may also open up new opportunities for wider applications. One possible direction is to explore proper physical constraints to enable omni-repulsion of individual magnetic microrobots such as using electrostatic double-layer forces, and create desired 3D potential energy landscapes.

\section{ACKNOWLEDGEMENT}

We thank W. Wang for helping characterize the contact angles of magnetic microrobots and A. C. Karacakol for helping characterize the magnetization of magnetic microrobots. The research is supported by the Max Planck Society. M.S. is also partially supported by the NSF, National Robotics Initiative program under grant number NRI-1317477. Supplementary videos is provided online by this link. 


\section{REFERENCES}

[1] Liu, C., Xu, T., Xu, L.P. and Zhang, X. Controllable swarming and assembly of micro/nanomachines. Micromachines, 9(1):10, 2017.

[2] Sitti, M. et al. Biomedical applications of untethered mobile milli/microrobots. Proc. IEEE, 103(2):205-224, 2015.

[3] Nelson, B. J., Kaliakatsos, I. K. and Abbott, J. J. Microrobots for minimally invasive medicine. Annu. Rev. Biomed. Eng. 12:55-85, 2010.

[4] Ceylan, H., Giltinan, J., Kozielski, K. and Sitti, M. Mobile microrobots for bioengineering applications. Lab Chip, 17(10):1705-1724, 2017.

[5] Alapan, Y., et al. Soft erythrocyte-based bacterial microswimmers for cargo delivery. Science Robotics, 3(17): eaar4423, 2018.

[6] Hu, W., Lum, G. Z., Mastrangeli, M. and Sitti, M. Smallscale soft-bodied robot with multimodal locomotion. Nature, 554(7690):81-85, 2018.

[7] Whitesides, G. M. and Grzybowski, B. Self-assembly at all scales. Science, 295(5564):2418-2421, 2002.

[8] Rubenstein, M., Cornejo, A. and Nagpal, R. Programmable self-assembly in a thousand-robot swarm. Science, 345(6198):795-799, 2014.

[9] Yang, G.Z. et al. The grand challenges of Science Robotics. Science Robotics, 3(14):eaal7650, 2018.

[10] Daudelin, J., Jing, G., Tosun, T., Yim, M., Kress-Gazit, H., and Campbell, M. An integrated system for perceptiondriven autonomy with modular robots. Science Robotics, 3(23): eaat4983, 2018.

[11] Chowdhury, S., Jing, W. and Cappelleri, D. J. Controlling multiple microrobots: recent progress and future challenges. Journal of Micro-Bio Robotics, 10(1-4):1-11, 2015.

[12] Diller, E., Floyd, S., Pawashe, C. and Sitti, M. Control of multiple heterogeneous magnetic microrobots in two dimensions on nonspecialized surfaces. IEEE Trans. Robot., 28(1):172-182, 2012.

[13] Diller, E., Giltinan, J. and Sitti, M. Independent control of multiple magnetic microrobots in three dimensions. Int. J. Robot. Res., 32(5):614-631, 2013.

[14] Frutiger, D. R., Vollmers, K., Kratochvil, B. E. and Nelson, B. J. Small, fast, and under control: wireless resonant magnetic micro-agents. Int. J. Robot. Res., 29(5):613-636, 2010.

[15] Rahmer, J., Stehning, C. and Gleich, B. Spatially selective remote magnetic actuation of identical helical micromachines. Science Robotics, 2(3):eaal2845, 2017.

[16] Pawashe, C., Floyd, S. and Sitti, M. Multiple magnetic microrobot control using electrostatic anchoring. Applied Physics Letters, 94(16):164108, 2009.

[17] Torres, N. A. and Popa, D. O. Cooperative control of multiple untethered magnetic microrobots using a single magnetic field source. Automation Science and Engineering (CASE), 2015 IEEE International Conference on, pages 1608-1613, 2015.
[18]Diller, E., Pawashe, C., Floyd, S. and Sitti, M. Assembly and disassembly of magnetic mobile micro-robots towards deterministic 2-D reconfigurable micro-systems. Int. J. Robot. Res., 30(14):1667-1680, 2011.

[19] Miyashita, S., Diller, E. and Sitti, M. Two-dimensional magnetic micro-module reconfigurations based on intermodular interactions. Int. J. Robot. Res., 32(5):591-613, 2013.

[20]Han, K. et al. Sequence-encoded colloidal origami and microbot assemblies from patchy magnetic cubes. Sci. Adv., 3(8):e1701108, 2017.

[21] Wang, W., Giltinan, J., Zakharchenko, S. and Sitti, M. Dynamic and programmable self-assembly of micro-rafts at the air-water interface. Sci. Adv., 3(5):e1602522, 2017.

[22] Servant, A., Qiu, F., Mazza, M., Kostarelos, K. and Nelson, B. J. Controlled in vivo swimming of a swarm of bacteria-like microrobotic flagella. Adv. Mater., 27(19):2981-2988, 2015.

[23] Wu, Z., et al. A swarm of slippery micropropellers penetrates the vitreous body of the eye. Sci. Adv., 4(11):eaat4388, 2018.

[24]Felfoul, O., et al. Magneto-aerotactic bacteria deliver drug-containing nanoliposomes to tumour hypoxic regions. Nature nanotechnology, 11(11):941, 2016.

[25] De Lanauze, et al. Three-dimensional remote aggregation and steering of magnetotactic bacteria microrobots for drug delivery applications. Int. J. Robot. Res., 33(3):359374, 2014.

[26] Snezhko, A. and Aranson, I. S. Magnetic manipulation of self-assembled colloidal asters. Nat. Mater., 10(9):698$703,2011$.

[27] Yu, J., et al. Ultra-extensible ribbon-like magnetic microswarm. Nat. Commun., 9(1):3260, 2018.

[28] Sumpter, D. J. T. Collective Animal Behavior (Princeton Univ. Press, 2010).

[29] Mlot, N. J., Tovey, C. A. and Hu, D. L. Fire ants selfassemble into waterproof rafts to survive floods. Proc. Natl Acad. Sci. USA, 108(19):7669-7673, 2011.

[30] Kummer, et al. OctoMag: An electromagnetic system for 5-DOF wireless micromanipulation. IEEE Trans. Robot., 26(6):1006-1017, 2010.

[31] Choi, J. S. and Yoo, J. Design of a Halbach magnet array based on optimization techniques. IEEE Trans. Magn., 44(10):2361-2366, 2008.

[32] Cooley, C. Z. et al. Design of sparse Halbach magnet arrays for portable MRI using a genetic algorithm. IEEE Trans. Magn., 54(1):1-12, 2018.

[33] Woodward, M. A. and Sitti, M. Universal custom complex magnetic spring design methodology. IEEE Trans. Magn., 54(1):1-13 (2018).

[34] Bertsimas, D. and Shioda, R. Classification and regression via integer optimization. Oper. Res., 55(2):252-271, 2007.

[35] Petruska, A. J. and Abbott, J. J. Optimal permanentmagnet geometries for dipole field approximation. IEEE Trans. Magn., 49(2):811-819, 2013.

[36] Kralchevsky, P.A. and Nagayama, K. Capillary interactions between particles bound to interfaces, liquid 
films and biomembranes. Adv. Colloid Interface Sci., 85(2-3):145-192, 2000.

[37] Brackenbury, J. Caterpillar kinematics. Nature, 390:453, 1997.

[38] Khatib, O. Real-time obstacle avoidance for manipulators and mobile robots. Int. J. Robot. Res., 5(1):90-98, 1986.

[39] Wang, et al. Collectives of Spinning Mobile Microrobots for Navigation and Object Manipulation at the Air-Water Interface. Intelligent Robots and Systems (IROS), 2018 IEEE/RSJ International Conference on, pages 1-8, 2018.

[40] Wang, Q., et al. Collective behavior of reconfigurable magnetic droplets via dynamic self-assembly. ACS Appl. Mater. Interfaces, 11(1):1630-1637, 2019.

[41] Shahrokhi, S., Lin, L., Ertel, C., Wan, M. and Becker, A. $\mathrm{T}$. Steering a swarm of particles using global inputs and swarm statistics. IEEE Trans. Robot., 34(1):207-219, 2017.

[42]Becker, et al. Massive uniform manipulation: Controlling large populations of simple robots with a common input signal. Intelligent Robots and Systems (IROS), 2013 IEEE/RSJ International Conference on, pages 520-527, 2013

[43] Tasoglu, S., et al. Guided and magnetic self-assembly of tunable magnetoceptive gels. Nat. Commun., 5:4702, 2014.

[44]Cao, Y., et al. Unconventional superconductivity in magic-angle graphene superlattices. Nature, 556:43-50, 2018. 